\title{
miR-140-5p inhibits the proliferation, migration and invasion of vascular smooth muscle cells by suppressing the expression of NCKAP1
}

\author{
Qing Ma, Jiancheng Liu, Chunbo Li, Dong Wang \\ Cardiovascular Surgery, Tangshan Gongren Hospital, Tangshan, Hebei Province, 063000, China
}

\begin{abstract}
Introduction. The occurrence of aortic dissection is related to the proliferation and metastasis of vascular smooth muscle cells. In our present study, we found that the expression of miR-140-5p was inhibited in the wall of abdominal aorta of aortic dissection patients. However, the mechanism of miR-140-5p in the development of aortic dissection is unclear.

Material and methods. We detected the expression of miR-140-5p and NCK Associated Protein 1 (NCKAP1) in blood vessel of aortic dissection patients and normal people by PCR. Next, we established the miR-140-5p overexpression and miR-140-5p inhibition vascular smooth muscle cells (CRL-1999 cells). The BrdU assays, wound healing assays and transwell assays were performed to detect the proliferation and invasion ability of these cells. Finally, luciferase reporter assay was performed to detect the relationship between miR-140-5p and NCKAP1. Results. The expression of miR-140-5p was suppressed in blood vessel of aortic dissection patients, and the levels of NCKAP1 in those tissues were upregulated. Overexpression of miR-140-5p inhibited the proliferation, migration and invasion of vascular smooth muscle cells. miR-140-5p targeted and suppressed the expression of NCKAP1. Conclusions. miR-140-5p repressed the proliferation, migration and invasion of vascular smooth muscle cells by targeting and inhibiting the expression of NCKAP1. Furthermore, the results of our study suggest new strategies and targets for the clinical treatment of arterial dissection. (Folia Histochemica et Cytobiologica 2021, Vol. 59, No. 1, 22-29)
\end{abstract}

Key words: aortic dissection; miR-140-5p; NCKAP1; vascular smooth muscle cells; proliferation; invasion

\section{Introduction}

Aortic dissection is a cardiovascular disease induced by blood entering the middle layer of the vessel wall and the formation of swelling [1]. Aortic dissection is a serious life-threatening cardiovascular disease. In addition to vascular endothelial damage, it can also Cause serious complications [2]. At present, the effective treatment of aortic dissection is mainly surgery. However, statistical data showed that the mortality rate of postoperative period is still 9-30\% [3]. Therefore,

Correspondence address: Dong Wang,

Cardiovascular Surgery, Tangshan Gongren Hospital, No. 27,

Wenhua Road, Tangshan City, Hebei Province, China

phone: 86+03152305089, e-mail: wangdongwd12345@163.com there is a need to determine the molecular mechanism of aortic dissection to develop the new therapeutic treatments. The application of drugs intervening in the disease's pathomechanism combined with surgery is expected to further improve the survival rate of patients. Furthermore, as the component of aortic vessel wall, the lesion of vascular smooth muscle cells (VSMCs) was considered as the important reasons for the onset of aortic dissection [4]. The VSMCs of aortic wall mainly showed low differentiation, strong proliferation and metastasis [5]. In addition, the large amount of extracellular matrix secreted by VSMCs also promotes their proliferation and invasion [6]. These effects of VSMCs attenuate the stability of the blood vessel wall, which in turn may induce the occurrence of aortic dissection [7]. 
Non-coding RNA is a type of RNA that does not translate into protein. Recent studies have found that non-coding RNA played a crucial role during the physiological process of cell differentiation, apoptosis, proliferation and metabolism $[8,9]$. Other research also revealed that miRNA was related to the occurrence and development of aortic dissection [10]. On the other hand, the overexpression of miRNA-30a promoted the development of aortic dissection [11]. Our results showed that levels of miR-140-5p were also downregulated in the VSMCs during the development of aortic dissection. However, the function of miR-140$5 p$ during the development of aortic dissection and its molecular mechanism are unclear. Therefore, we explored the effect of miR-140-5p on the development of aortic dissection in an in the culture of VSMCs. Our findings suggest that miR-140-5p has the potential to suppress the aortic dissection by restricting the proliferation and metastasis of vascular smooth muscle cells.

\section{Material and methods}

Collection of tissue samples. Samples of ascending aorta tissue (10 samples of aortic dissection patients and 7 samples of normal people, all these patients were 35 years old, half of the patients was male and others were female, 3 normal people were male and others were female) were collected from the hospital and used for the detection. These samples were collected during the clinical operation. Then we used liquid nitrogen to freeze these samples, and then we used a mortar to grind these samples. Finally, Trizol (Thermo Fisher Scientific, Waltham, MA, USA) was applied to extract RNA and protein from these samples. This experiment has been approved by the Ethics Committee of Tangshan Gongren Hospital, Tangshan, China. All the operation of this study followed the World Medical Association Declaration of Helsinki [12].

Cell culture and transfection. Human aortic vascular smooth muscle cell line (CRL-1999) was applied for the experiments in this study. CRL-1999 cell line was acquired from the ATCC (Manassas, VA, USA). All the cells were incubated in $37^{\circ} \mathrm{C}$ humid atmosphere with $5 \% \mathrm{CO}_{2}$ in RPMI-1640 medium (Hyclone, Logan, UT, USA) supplemented with the $10 \%$ fetal bovine serum (FBS, Gibco, Gaithersburg, MD, USA). Furthermore, the inhibitor and mimic of miR-140-5p were obtained from the Genechem (Shanghai, China) and applied for the transfection of these cells. Plasmids used in this assay were obtained from the Genechem. Polybrene was mixed with the lentivirus (with the ratio of 1:7) containing the inhibitor and mimic of miR-140-5p and applied to promote the efficiency of transfection. Subsequently, the mixed lentivirus medium was co-cultured with these cells for 12 hours to complete the transfection. Since the plasmid vector contained antibiotic resistance genes, we used media supplemented with puromycin $(1 \mathrm{mg} / \mathrm{mL}$, Thermo Fisher Scientific) to culture these cells in subsequent experiments to screen for successfully transfected cells.

Bromodeoxyuridine (BrdU) incorporation assay. CRL-1999 cells were incubated with $10 \mu \mathrm{m}$ bromodeoxyuridine (BrdU) (Sigma, Burbank CA, USA) for 4 hours in the incubator. Next, these cells were rinsed with the PBS buffer (phosphate-buffered saline) and fixed with $4 \%$ formaldehyde. Then, these cells were washed with PBS again and incubated with the $4 \mathrm{~N} \mathrm{HCl}$ containing $1 \%$ Triton X-100 for 15 min. Next, anti-BrdU antibodies (SigmaAldrich) were diluted with PBS and incubated with the cells. At last, these cells were incubated with anti-mouse IgG-Cy3 (SigmaAldrich) in the dark and the fluorescence was detected by spectrophotometer (Aoxi Company, Shanghai, China).

Cell counting kit-8 (CCK-8). Before the assays, CRL-1999 cells were plated into four 96 well plates. After the adhesion (0, 24, 48 and 72 hours) of these cells, the CCK-8 (Dojindo, Kumamoto, Japan) was diluted with the RPMI-medium (1:10) and incubated with the cells at $37^{\circ} \mathrm{C}$ for 1 hour. Then, the absorbance of these cells was detected by spectrophotometer (Aoxi Company). And the absorbance of these cells reflected the proliferation of these cells.

Wound healing assays. CRL-1999 cells were plated in the 6 well plates before the experiment. After adhesion was reached, the cells were cultured with the serum-free medium for $12 \mathrm{~h}$. Then, the scratch was created with the pipette tip. The scratch was photographed with inverted microscope (Olympus, Tokyo, Japan) after 0 and $24 \mathrm{~h}$. The width of the scratch was measured with the Image $\mathrm{J}$ software (National Institutes of Health, Bethesda, MD, USA).

Transwell assay. CRL-1999 cell line was cultured with the medium without FBS serum for $12 \mathrm{~h}$. In addition, the matrix gel (10 mg/mL, BD, USA) was diluted with the FBS-free medium (with the ratio of 1:10) and added into the upper layer of the $8 \mu \mathrm{m}$ Boyden chamber (Corning, NY, USA). Cells were seeded into the upper layer of the chamber. The medium containing FBS was added into the lower layer of the chamber. Then, Boyden chamber was placed in the incubator for $24 \mathrm{~h}$. Then, cells that pass through the aperture were stained with the crystal violet (Thermo Fisher Scientific) and photographed with inverted microscope.

RT-PCR. Total RNA was collected with the Trizol (Thermo Fisher Scientific). Then, the reverse transcription kit (Takara, Shiga, Japan) was used for the reverse transcription of RNA. Next, ABI7500 system (Thermo Fisher Scientific) was used for the amplification of cDNA and the relative levels of the target genes were analyzed with the $2^{-\Delta \Delta \mathrm{Ct}}$ method [13]. The primers used in this study were listed in Table 1. 
Table 1. Primers used for the qPCR

\begin{tabular}{|l|c|c|}
\hline Gene & Forward primer & \multicolumn{1}{c|}{ Reverse primer } \\
\hline miR-140-5p & 5'-TGCGGCAGTGGTTTTACCCTATG-3' & 5'-CCAGTGCAGGGTCCGAGGT-3' \\
\hline U6 & 5'-TGCGGGTGCTCGCTTCGGCAGC-3' & 5'-CCAGTGCAGGGTCCGAGGT-3' \\
\hline
\end{tabular}

Western blotting. Total protein was extracted by RIPA buffer (Beyotime, Beijng, China). Then, protein concentration was determined by BCA method (Beyotime). After that, proteins were segregated by $10 \%$ SDS-PAGE gel (Beyotime). Next, PVDF membranes were used for the adsorption of these proteins. Then, $5 \%$ skim milk powder (BD, USA) was prepared for the blocking of these membranes. After that, these membranes were hatched by primary antibodies. The primary antibodies used in this research included MMP-2 (Abcam, ab51075), MMP-9 (Abcam, ab76003), NCKAP1 (Abcam, ab126061) and $\beta$-actin (Abcam, ab8226). In the second day, these membranes were hatched with the second antibody (Abcam, ab6721). These primary and second antibodies were diluted with the antibody diluent (Beyotime) with the ratio (1:1000). Finally, the bands were emerged with the enhanced chemiluminescence reagents (Pierce, Rockford, IL, USA).

Luciferase reporter assays. CRL-1999 cells were cultured in the 6 well plates. Next, psiCHECKTM-2 vector (Genechem) contained the cloned miR-140-5p binding site of NCKAP1 (NCKAP1-wild type) was co-transfected with mimics NC or miR-140-5p mimics by Lipofectamine ${ }^{2000}$ transfection reagent. The psiCHECKTM-2 vector containing a mutant sequence of miR-140-5p binding site (3'-untranslated region of NCKAP1), named NCKAP1-mutation (Mut), was established with QuickChange Site-Directed Mutagenesis Kit (Stratagene, La Jola, CA, USA). Fluorescence intensity was detected with the luciferase Reporter Assay Kit (Promega, Fitchburg, WI, USA) after the transfection.

Statistical analysis. GraphPad Prism 6.0 software (GraphPad Software Inc., San Diego, CA, USA) was used for analyzing of the data. And data was displayed as mean \pm standard deviation (SD) in this paper. All the experiments were repeated for three times. The linear regression was used for the analysis of the expression between miR-140-5p and NCKAP1. Student's $t$ test was applied for the analyzed of the data in this paper. And there is the statistically significant difference between the two groups until the values of $p$ was less than 0.05 .

\section{Results}

\section{miR-140-5p inhibited proliferation of vascular smooth muscle cells}

To detect the efficacy of miR-140-5p on development of aortic dissection, we detected the expression of miR-140-5p in blood vessel wall of aortic dissection

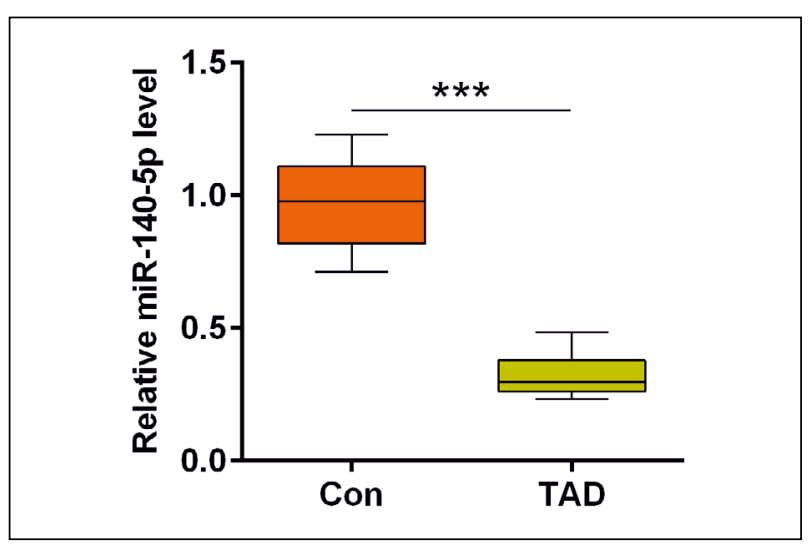

Figure 1. The expression of miR-140-5p was inhibited in blood vessel tissues of aortic dissection patients. The expression of miR-140-5p in vessel tissues of aortic dissection patients and normal vessel tissues was detected with the RT-PCR as described in Methods. Con, tissue from aorta's wall of normal subjects; TAD, tissue from the wall of aortic dissection patients. ${ }^{* *} p<0.001$.

patients and normal vascular tissues. According to the results (Fig. 1), we revealed that the levels of miR-140-5p were decreased during the occurrence and development of aortic dissection. Next, lentivirus contained the plasmids of overexpression miR-140-5p or miR-140-5p shRNA were applied to upregulate or downregulate miR-140-5p in CRL-1999 cells, respectively. Then the CCK-8 assay was applied for the detection of the viability of these cells. The results in Figure 2A showed that the viability of these cells was repressed after the overexpression of miR-140-5p and enhanced after the inhibition of miR-140-5p. Similarly, results (Fig. 2B) of BrdU assays also showed that overexpression of miR-140-5p suppressed the proliferation of these cells. Furthermore, the inhibition of miR-140-5p promoted the proliferation of these cells. Results of this part revealed that miR-140-5p suppressed the proliferation and viability of vascular smooth muscle cells.

\section{miR-140-5p suppressed the migration and invasion of vascular smooth muscle cells}

Next, we detected the migration of CRL-1999 cells by wound healing assays. The results (Fig. 3A) showed that the healing rate of scratches was slower when the expression of miR-140-5p was promoted in these cells. However, the healing rate was faster when the levels 


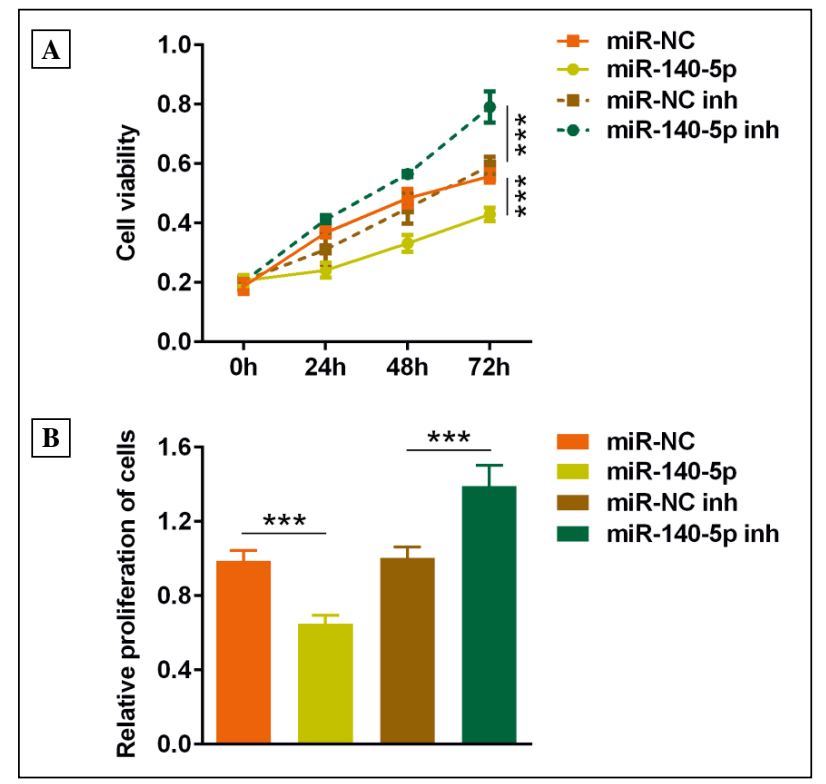

Figure 2. Overe xpression of miR-140-5p inhibited the proliferation of vascular smooth muscle cells. A. The cell viability of vascular smooth muscle cells was detected with the CCK-8 assay. B. Proliferation of vascular smooth muscle cells was determined with BrdU assay. ${ }^{* *} p<0.001$. of miR-140-5p were inhibited. In addition, results (Fig. 3B) of transwell showed that the quantity of invasion cells was reduced after overexpression of miR-140-5p. However, the number of invasion cells was promoted after inhibition of miR-140-5p in CRL-1999 cells. Furthermore, the results (Fig. 3C) of western blotting showed that the expression of MMP-2 and MMP-9 was inhibited after overexpression of miR-140-5p. However, the levels of MMP-2 and MMP-9 were upregulated after inhibition of miR-140-5p. And these results suggested that miR$140-5 p$ restricted the migration and invasion of vascular smooth muscle cells.

\section{miR-140-5p targeted and restricted the expression of NCKAP1 in vascular smooth muscle cells}

By querying Targetscan database (targetscan, http:// www.targetscan.org/vert 72/), we found that miR140-5p had the potential to bind to the 3'-UTR of NCKAP1. The results (Fig. 4A) of RT-PCR also showed that the expression of NCKAP1 was promoted in blood vessel wall of aortic dissection patients compared to the normal tissues. And the results (Fig. 4B)

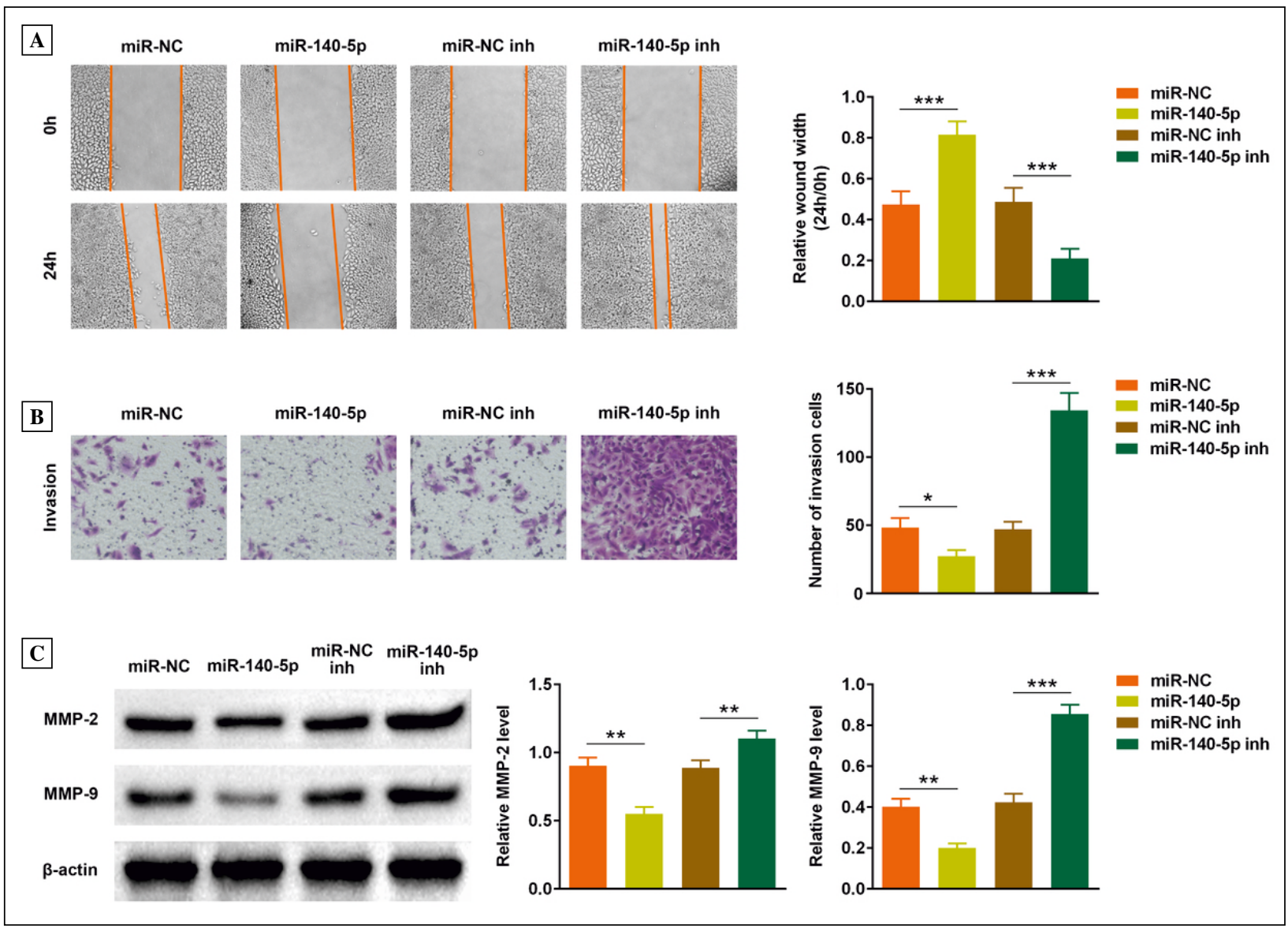

Figure 3. Overexpression of miR-140-5p inhibited the migration and invasion of vascular smooth muscle cells. A. The migration of vascular smooth muscle cells was detected by the wound healing assay. B. Invasion of vascular smooth muscle cells was determined with transwell assay. C. The expression of MMP-2 and MMP-9 were detected by western blotting. ${ }^{*} p<0.05,{ }^{* *} p<0.01,{ }^{* * *} p<0.001$. 


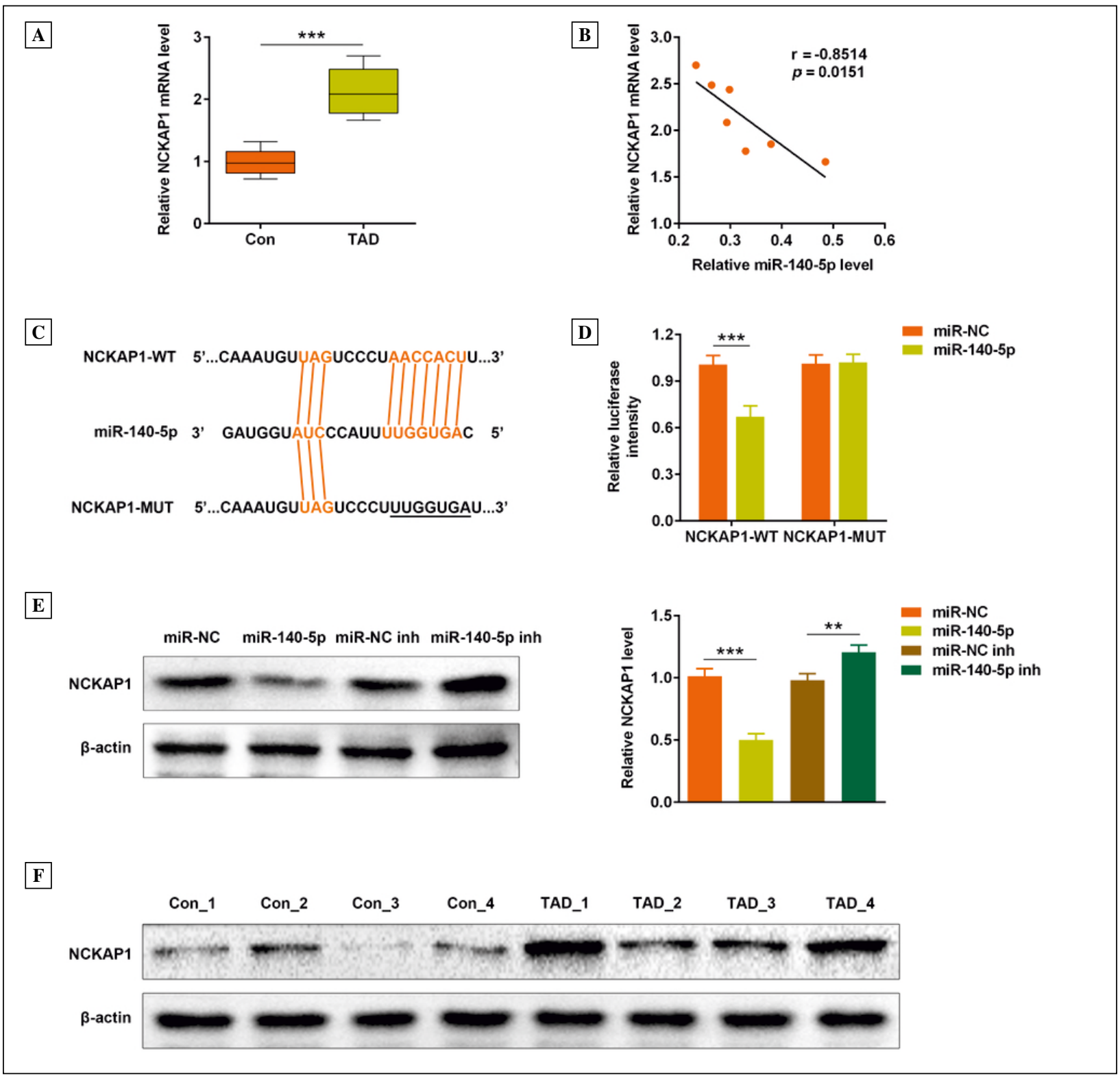

Figure 4. miR-140-5p targeted and inhibited the expression of NCKAP1 in vascular smooth muscle cells. A. The expression of NCKAP1 in clinical samples was detected with the RT-PCR. B. Linear regression analysis was performed to detect the relationship between miR-140-5p and NCKAP1. C. Target region between miR-140-5p and NCKAP1 was depicted. D. The fluorescence intensity of vascular smooth muscle cells was measured with the spectrophotometer. E. Western blotting was performed to detect the expression of NCKAP1 in vascular smooth muscle cells after the overexpression or inhibition of miR-140-5p. F. The expression of NCKAP1 in vessel tissues of aortic dissection patients and normal vessel tissues was detected with western blotting. ${ }^{* *} p<0.01,{ }^{* * *} p<0.001$.

of linear-regression analysis also showed that the expression of NCKAP1 was negatively correlated with the levels of miR-140-5p. Results of luciferase reporter assay also showed that the fluorescence intensity was suppressed in the NCKAP1 wild type and miR140-5p overexpression system. However, there was no difference of the fluorescence between the negative control and miR-140-5p overexpression group after the mutation of 3'-UTR of NCKAP1 (Fig. 4C and Fig.
4D). Next, the expression of NCKAP1 in miR-140-5p overexpression and suppression CRL-1999 cells was determined by western blotting. The results (Fig. 4E) revealed that the level of NCKAP1 was repressed when the expression of miR-140-5p was promoted in these cells. Moreover, expression of NCKAP1 was enhanced when the expression of miR-140-5p was suppressed in CRL-1999 cells. Finally, we also found that the expression of NCKAP1 was upregulated in 


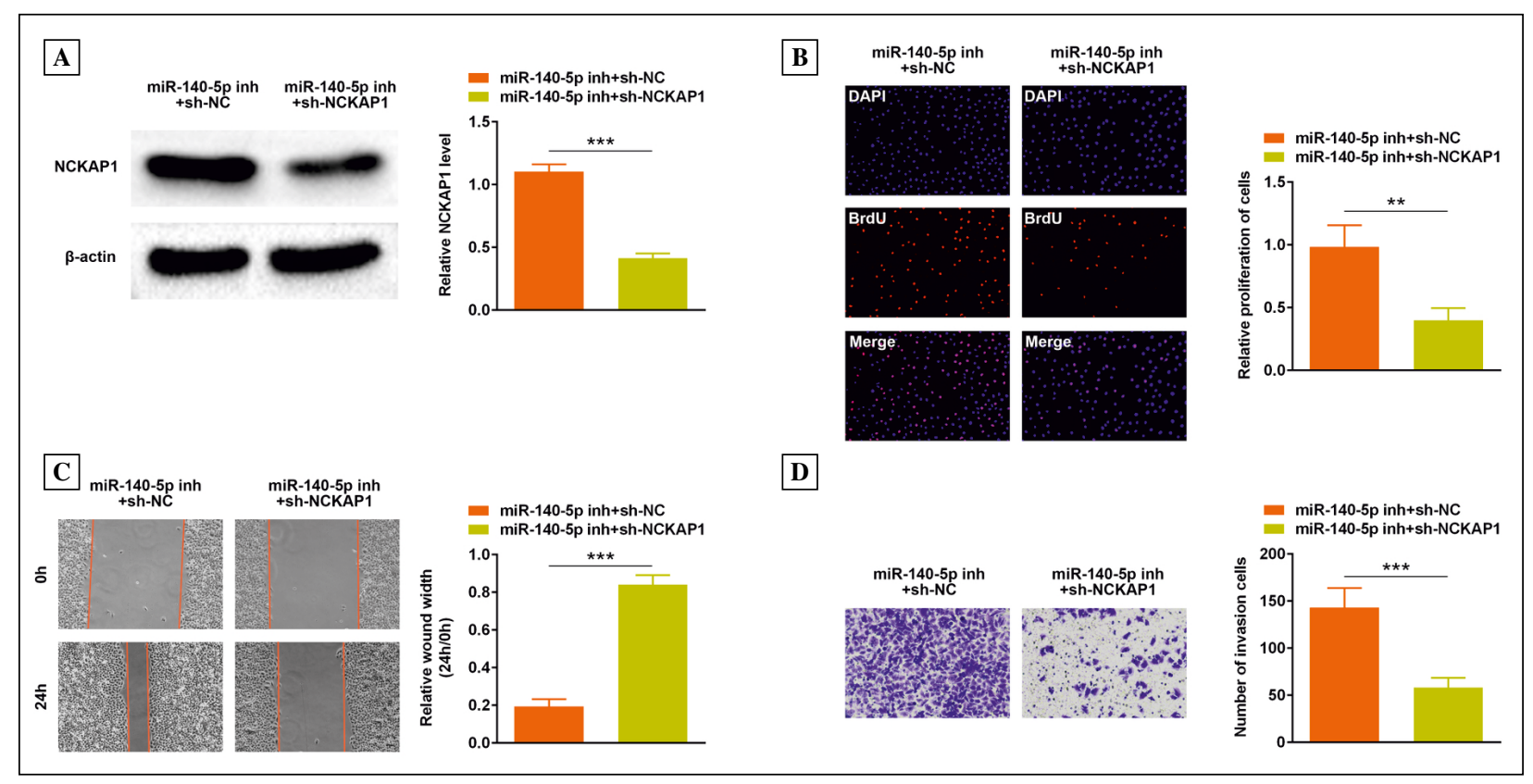

Figure 5. Suppression of NCKAP1 repressed the proliferation, migration and invasion of miR-140-5p inhibition vascular smooth muscle cells. A. The expression of NCKAP1 was detected by western blotting. B. BrdU assay was performed to detect the proliferation of vascular smooth muscle cells. C. Wound healing assay was conducted to detect the migration of vascular smooth muscle cells. D. Invasion of vascular smooth muscle cells was determined with the transwell assay. $* * p<0.01, * * * p<0.001$.

arterial vascular wall of aortic dissection patients compared to the normal tissues (Fig. 4F).

\section{Repression of NCKAP1 restricted the proliferation, migration and invasion of miR-140-5p inhibition vascular smooth muscle cells}

In this part, we suppressed the expression of NCKAP1 in miR-140-5p inhibition vascular smooth muscle cells. The results (Fig. 5A) showed that the protein levels of NCKAP1 were decreased in the cells transfected with sh-NCKAP1. And the results (Fig. 5B) of BrdU assays showed that the proliferation of CRL-1999 cells was inhibited after the suppression of NCKAP1. Finally, wound healing and transwell assays were performed to explore the changing of migration and invasion of CRL-1999 cells after the repression of NCKAP1. According to the results (Fig. 5C and Fig. 5D), the migration and invasion of the miR-140-5p inhibition CRL-1999 cells were inhibited after the suppression of NCKAP1. These results also implied that the effects of miR-140-5p/NCKAP1 axis on the proliferation, migration and invasion of vascular smooth cells.

\section{Discussion}

Weakening of the arterial wall often induced the formation of aortic dissection. And the main feature of aortic dissection is the separation of the aortic media induced by blood flow [14]. And the development of aortic dissection may induce the occurrence of the aortic aneurysm [15]. However, the specific molecular mechanism of aortic dissection is still unclear.

Furthermore, vascular smooth muscle cell is the main cell type in the media layer of the aorta. And the existent of vascular smooth muscle cell played an important role in maintaining the normal physiological function of the aortic wall $[1,16]$. In addition, previous research revealed that the EZH 2 could affect the development of aortic dissection by regulating the autophagy of vascular smooth muscle cells [17]. Some studies also suggested that proliferation and metastasis of vascular smooth muscle cells were correlated to the development of vascular disease [18, 19]. For instance, miR-146a-5p promoted the development of aortic dissection by enhancing the proliferation and migration of vascular smooth muscle cells [20]. However, there is also research revealed that the proliferation of VSMCs led to the occurrence of plaque of blood vessels and did not induce the aortic dissection [21]. Most of these results from the previous studies suggested that the intensive proliferation and migration of vascular smooth muscle cell promoted the occurrence and development of aortic dissection. In addition, some studies also pointed out that miR-140-5p repressed the proliferation of human pulmonary artery smooth muscle cells $[22,23]$. Similarly, we also found that the expression 
of miR-140-5p was downregulated in arterial wall of aortic dissection patients. In addition, overexpression of miR-140-5p repressed the proliferation and migration of vascular smooth muscle cells. All these results suggested that miR-140-5p restricted the proliferation, migration and invasion of vascular smooth muscle cell. And the results of our study implied that higher levels of miR-140-5p might have the potential to repress the development of the aortic dissection by suppressing the proliferation, migration and invasion of vascular smooth muscle cells.

On the other hand, miRNAs could affect the expression of target genes by binding to the 3'-UTR of the these genes [24]. By querying the database, we found that miR-140-5p has potential to bind to and affect the expression of NCKAP1. NCKAP1 is localized along the lamellipodia and associated with the migration of multiple types of cells [25]. Another study also revealed that the expression of NCKAP1 promoted the metastasis of breast tumor cells and induced poor prognosis of these patients [26]. Previous study suggested that miR-214 repressed the proliferation, migration and invasion of vascular smooth muscle cells by inhibiting the expression of NCKAP1. In this study, we revealed that miR-140-5p targeted and suppressed the expression of NCKAP1. And the expression of NCKAP1 was also upregulated in the arterial wall of aortic dissection patients. Furthermore, repression of NCKAP1 restricted the proliferation, migration and invasion of miR-140-5p inhibition vascular smooth muscle cells. These results also indicated that NCKAP1 was played a crucial role during the development of aortic dissection. And the results in our study suggested that NCKAP1 can enhance the proliferation and invasion of multiple types of cells. Above all, in this study, we revealed that miR-140-5p inhibited the proliferation, migration and invasion of vascular smooth muscle cells by decreasing of the levels of NCKAP1. And results also implied that miR-140-5p has the potential to impede the development of aortic dissection. Moreover, the results of our research offered new targets and strategy of the clinic treatment of aortic dissection.

\section{Acknowledgements}

Not applicable.

\section{Funding}

Not applicable.

\section{Competing interests}

The authors state that there are no conflicts of interest to disclose.

\section{Ethics approval}

Ethical approval was obtained from the Ethics Committee of Tangshan Gongren Hospital.

\section{Statement of Informed Consent}

Written informed consent was obtained from a legally authorized representative(s) for anonymized patient information to be published in this article.

\section{Availability of data and materials}

All data generated or analyzed during this study are included in this published article.

\section{Authors' contributions}

Qing Ma and Jiancheng Liu designed the study, supervised the data collection, Chunbo Li analyzed the data, interpreted the data, Dong Wang prepare the manuscript for publication and reviewed the draft of the manuscript. All authors have read and approved the manuscript.

\section{References}

1. Golledge J, Eagle K. Acute aortic dissection. Lancet. 2008; 372(9632): 55-66, doi: 10.1016/s0140-6736(08)60994-0, indexed in Pubmed: PMID: 18603160.

2. Parthenakis F, Koutalas E, Patrianakos A, et al. Diagnosing acute aortic syndromes: The role of specific biochemical markers. Int J Cardiol. 2010; 145(1): 3-8, doi: 10.1016/j.ijcard.2010.04.022, indexed in Pubmed: 20483494.

3. Pagni S, Ganzel B, Trivedi J, et al. Early and midterm outcomes following surgery for acute type A aortic dissection. J Card Surg. 2013; 28(5): 543-549, doi: 10.1111/jocs.12170, indexed in Pubmed: 23909254.

4. Mészáros I, Mórocz J, Szlávi J, et al. Epidemiology and clinicopathology of aortic dissection. Chest. 2000; 117(5): 1271-1278, doi: 10.1378/chest.117.5.1271, indexed in Pubmed: 10807810.

5. Wei X, Sun Y, Wu Y, et al. Downregulation of Talin-1 expression associates with increased proliferation and migration of vascular smooth muscle cells in aortic dissection, BMC Cardiovasc Disord. 2017; 17: 162, doi: 10.1186/s12872-017-0588-0, indexed in Pubmed: 28637452.

6. Hao H, Gabbiani G, Bochaton-Piallat ML. Arterial smooth muscle cell heterogeneity. Arterioscler Thromb Vasc Biol. 2003; 23(9): 1510-1520, doi: 10.1161/01.atv.0000090130.85752. ed, indexed in Pubmed: PMID: 12907463.

7. Salabei J, Hill B. Implications of autophagy for vascular smooth muscle cell function and plasticity. Free Radic Biol Med. 2013; 65: 693-703, doi: 10.1016/j.freeradbiomed.2013.08.003, indexed in Pubmed: 23938401.

8. Jin YY, Wang JF, Wang XJ, et al. Roles of non-coding RNA in pancreatic islet development and functioning. Zhongguo Yi Xue Ke Xue Yuan Xue Bao. 2014; 36: 691-696, doi: 10.3881/j. issn.1000-503X.2014.06.025, indexed in Pubmed: 25556748.

9. Schwarzer A, Emmrich S, Schmidt F, et al. The non-coding RNA landscape of human hematopoiesis and leukemia, Nat Commun. 2017; 8 (1): 218, doi: 10.1038/s41467-017-00212-4, indexed in Pubmed: 28794406. 
10. Wang Y, Dong CQ, Peng GY, et al. MicroRNA-134-5p Regulates media degeneration through inhibiting VSMC phenotypic switch and migration in thoracic aortic dissection. Molecular Therapy - Nucleic Acids. 2019; 16: 284-294, doi: 10.1016/j.omtn.2019.02.021, indexed in Pubmed: 30951965.

11. Yu Y, Shi E, Gu T, et al. Overexpression of microRNA-30a contributes to the development of aortic dissection by targeting lysyl oxidase. J Thorac Cardiovasc Surg. 2017; 154(6): 1862-1869, doi: 10.1016/j.jtcvs.2017.06.019, indexed in Pubmed: 28711328.

12. World Medical Association Declaration of Helsinki: ethical principles for medical research involving human subjects. J Am Coll Dent. 2014; 81: 14-18, indexed in Pubmed: 25951678.

13. Walton T, Li G, McCulloch T, et al. Quantitative RT-PCR analysis of estrogen receptor gene expression in laser microdissected prostate cancer tissue. Prostate. 2009; 69(8): 810-819, doi: 10.1002/pros.20929, indexed in Pubmed: 19189301.

14. Takeda N, Komuro I. Genetic basis of hereditary thoracic aortic aneurysms and dissections. J Cardiol. 2019; 74(2): 136-143, doi: 10.1016/j.jjcc.2019.03.014, indexed in Pubmed: 31000321.

15. Martino ADe, Morganti R, Falcetta G, et al. Acute aortic dissection and pregnancy: Review and meta analysis of incidence, presentation, and pathologic substrates. J Card Surg. 2019; 34(12): 1591-1597, doi: 10.1111/jocs.14305, indexed in Pubmed: 31794127.

16. Barbour J, Spinale F, Ikonomidis J. Proteinase systems and thoracic aortic aneurysm progression. J Surg Res. 2007; 139(2): 292-307, doi: 10.1016/j.jss.2006.09.020, indexed in Pubmed: 17292415.

17. Li R, Yi X, Wei X, et al. EZH2 inhibits autophagic cell death of aortic vascular smooth muscle cells to affect aortic dissection. Cell Death Dis. 2018; 9: 180, doi: 10.1038/s41419-0170213-2, indexed in Pubmed: 29416002.

18. Choi M, Lee I, Kim G, et al. Regulation of PDGF signalling and vascular remodelling by peroxiredoxin II. Nature. 2005;
435(7040): 347-353, doi: 10.1038/nature03587, indexed in Pubmed: 15902258.

19. Hopkins P. Molecular biology of atherosclerosis. Physiol Rev. 2013; 93(3): 1317-1542, doi: 10.1152/physrev.00004.2012, indexed in Pubmed: 23899566.

20. Xue L, Luo S, Ding H, et al. Upregulation of miR-146a-5p is associated with increased proliferation and migration of vascular smooth muscle cells in aortic dissection. J Clin Lab Anal. 2019; 33: e22843, doi: 10.1002/jcla.22843, indexed in Pubmed: 30779466.

21. Clément M, Chappell J, Raffort J, et al. Vascular smooth muscle cell plasticity and autophagy in dissecting aortic aneurysms. Arterioscler Thromb Vasc Biol. 2019; 39(6): 1149-1159, doi: 10.1161/atvbaha.118.311727, indexed in Pubmed: 30943775.

22. Rothman A, Arnold N, Pickworth J, et al. MicroRNA-140-5p and SMURF1 regulate pulmonary arterial hypertension. J Clin Invest. 2016; 126(7): 2495-2508, doi: 10.1172/jci83361, indexed in Pubmed: 27214554.

23. Zhang Y, Xu J. miR-140-5p regulates hypoxia-mediated human pulmonary artery smooth muscle cell proliferation, apoptosis and differentiation by targeting Dnmt1 and promoting SOD2 expression. Biochem Biophys Res Commun. 2016; 473(1): 342-348, doi: 10.1016/j.bbrc.2016.03.116, indexed in Pubmed: 27021683.

24. Loginov VI, Rykov SV, Fridman MV, et al. Methylation of miRNA genes and oncogenesis. Biochemistry (Moscow). 2015; 80(2): 145-162, doi: 10.1134/s0006297915020029, indexed in Pubmed: 25756530.

25. Nakao S, Platek A, Hirano S, et al. Contact-dependent promotion of cell migration by the OL-protocadherin-Nap1 interaction. J Cell Biol. 2008; 182(2): 395-410, doi: 10.1083/ jcb.200802069, indexed in Pubmed: 18644894.

26. Lomakina M, Lallemand F, Vacher S, et al. Arpin downregulation in breast cancer is associated with poor prognosis. $\mathrm{Br}$ J Cancer. 2016; 114(5): 545-553, doi: 10.1038/bjc.2016.18, indexed in Pubmed: 26867158.

Submitted: 21 September, 2020 Accepted after reviews: 28 January, 2021 Available as AoP: 9 February, 2021 